\title{
AN OPERATIONAL PLANNING MODEL FOR THE DYNAMIC VEHICLE ALLOCATION PROBLEM WITH UNCERTAIN DEMANDS $\dagger$
}

\author{
WARREN B. POWELL \\ Department of Civil Engineering, Princeton University, Princeton, New Jersey 08544, U.S.A.
}

(Received 17 January 1986; in revised form 10 June 1986)

\begin{abstract}
The dynamic vehicle allocation problem arises when a motor carrier must simultaneously and in real time coordinate the dispatching of vehicles from one region to the next all over the country. The decision to send a vehicle loaded or empty from one region to the next, arriving in the destination region at some point in the future, must anticipate the downstream impacts of the decision. The consequences of another load in a particular region at some point in the future, however, are highly uncertain. A simple methodology is proposed which calculates approximately the marginal value of an additional vehicle in each region in the future. This information is then used to generate a standard pure network which can be efficiently optimized to give dispatching decisions for today.
\end{abstract}

The dynamic vehicle allocation problem arises when a carrier must manage the positioning of a fleet of vehicles over time. The application motivating this paper arises from truckload motor carriers where a shipper might request a trailer to carry a load of freight from one city to the next on a given day. The shipper pays for the entire tractor-trailer combination and there is no issue of consolidating shipments on one trailer. The problem the carrier faces is one of anticipating the needs of shippers by ensuring that trailers are in the right place at the right time.

The principal difficulty is that a carrier typically has very little advance notice regarding future needs of a shipper. At the beginning of a day, a truckload carrier might only know $40 \%$ of the loads that will be carried that day (the remaining $60 \%$ are called in during the morning and early afternoon) and less than $20 \%$ of the loads that need to be carried the following day. Just the same, the carrier must be able to anticipate the future cost and revenue implications of decisions made now in order to make intelligent decisions regarding which loads the carrier should accept and where to move empty trailers.

The vehicle allocation problem was originally formulated as a transportation problem by considering current supplies of and demands for vehicles, and ignoring downstream impacts of decisions (see, for example, Misra, 1972). White and Bomberault (1969) and White (1972) indicated how the problem might be approached as a dynamic problem by setting up a network where each node represented a particular region at a particular point in time. Future demands for vehicles between regions would be forecasted over a specified planning horizon. If each forecast is assumed to be known with certainty, the problem reduces to a simple network transshipment problem. Such an approach is currently in use at one of the major truckload motor carrier (Crowe, 1983). A major drawback of the deterministic model, however, is that the forecasts must be integer in order to guarantee an integer solution. In many instances, and at almost every truckload motor carrier, demand forecasts are fractions in the range .05 to .5 , necessitating the use of heuristic rounding rules.

Recognizing the frequently large uncertainty associated with the demand forecasts, Powell et al. (1984) reformulated the problem as a nonlinear network by representing forecasts as random variables with known means and variances. The decision variables are still the number of vehicles moving between pairs of regions over time, but now the number of vehicles moving full is random, and the expected number of full vehicles, and hence the expected net revenue generated between every pair of regions, becomes a nonlinear function of the flow. A key assumption made that significantly simplifies the problem is that the number of vehicles moving from region $i$ to region $j$ on a given day is determined before the number of loads available is

tThis research was supported in part by the National Science Foundation under Grant ECE-8408044. 
known. This assumption implies that the flow of vehicles between regions is known deterministically. In reality, a carrier will often only move a vehicle if it can be moved full.

A more realistic model is described in Powell (1986) which distinguishes two types of vehicles moving between regions: vehicles that will only move if they can move full, and vehicles moving empty. If the number of vehicles that are supposed to move full in a given lane exceeds the actual demand, then the number of vehicles actually moving full is assumed to exactly equal the number of available loads, and the remaining vehicles are assumed to be held in that region until the next time period. A direct implication of this assumption is that the number of vehicles actually moving is random, and hence the supplies of empty vehicles in future time periods are also random.

In reality, even this last model is not very accurate since if a vehicle in region $i$ cannot be moved full to a particular region, it may be possible to move it full to another region. The standard network framework of assigning vehicles to the links of a network, which implicitly assigns a vehicle to a particular movement between regions, is not useful when a vehicle might possibly be used on any one of a number of possible links, depending on the loads that are actually available at that time. A second limitation of the nonlinear models is that the optimal solutions are not integer, limiting their use as a real-time vehicle routing tool.

This paper describes an alternative model which can be used in a real-time environment for determining how to manage a fleet of vehicles today while anticipating the consequences of decisions made now. The goal of the paper is to describe the mechanics of a relatively simple approach for handling real-time dispatching under uncertainty; readers familiar with the theory of Markov processes in general and Markovian decision theory in particular will recognize the problem as an interesting and challenging application of this theory. Important features of the approach are that it is computationally very fast and returns integer solutions which can be directly implemented. Several approximations, however, are made and it is important that these be fully understood before applying the approach. Section 1 provides a somewhat simplified overview of the overall network structure. A key component is the representation of what will happen to a vehicle once it arrives in a region at some time in the future given uncertain outbound loads from this region. The estimates of expected costs and revenues from a vehicle arriving in a region are calculated using the regional impact model, described in Section 2 . The regional impact model depends on the calculation of the dispatch probabilities outlined in Section 3 . The calculation of the regional impacts and the dispatch probabilities also depends on an estimate of the average value one more vehicle in a region will contribute from a particular point in time until the end of the planning horizon. These average values, termed regional end effects, are given in Section 4. Section 5 then reviews the basic network structure given in Section 1, pointing out how it can be easily generalized to handle "known loads" (loads which shippers have already called in) at future points in time. Next, Section 6 describes how forecasts of future supplies of vehicles can be estimated several days into the future, after which Section 7 discusses forecasting errors that are made by the model and steps that can be taken to correct them.

\section{THE NETWORK STRUCTURE: A FIRST CUT}

For pedagogical purposes, assume that the following data is available.

(i) Current known loads outbound from each region today to all other regions.

(ii) Current supplies of available vehicles, as well as vehicles which are currently moving loaded or empty but will become available at some time in the future.

(iii) The probability distribution of the number of loads that are forecast to be carried between every pair of regions on each day until the end of the planning horizon. This distribution is assumed to have been modified to reflect any loads that have already been called in.

(iv) The distribution of the number of empties that have historically been moved between every pair of regions on each day until the end of the planning horizon.

(v) The average net revenue per loaded move, and the average cost per empty move, as well as the travel times, between every pair of regions. 
The problem is posed as follows. Given the above data, we know what loads are available to be carried outbound from each region today, and we know how many vehicles we have to handle these loads. The decisions that must be made immediately are which of the available loads should be accepted (and which rejected) given available capacity, and where should unassigned empty vehicles be sent (they may simply be held). The decision to send a vehicle (loaded or empty) from region $A$ to region $B$ should take into account not just the net revenue derived from moving to $B$ but also the marginal contribution of that vehicle once it arrives to $B$. In addition, the marginal contribution of a vehicle sent to $B$ must reflect how many other vehicles have already been sent to $B$ and will arrive on the same day.

The problem can be approached by viewing the future trajectory of a vehicle as comprising three components (a) the initial dispatch of the vehicle (to move loaded or empty to another region, or to be held in the same region), (b) the second dispatch of the vehicle to some uncertain load, and (c) the set of movements beginning with the third dispatch of the vehicle until it reaches the end of the planning horizon. The problem is best visualized using Fig. 1, which illustrates the problem of evaluating the impact of sending a vehicle loaded from region 1 to region 2 , a movement depicted using a single solid line. Once in region 2, the range of possible outbound dispatches, which are uncertain when the vehicle was in region 1 , is shown as a set of dashed lines to various regions around the country. Each of these moves outbound from region 2 also features a range of possible dispatches after arriving in the destination region, depicted as a series of white outlined arrows.

The optimization problem can be set up as a network as shown in Fig. 2, which consists of two key types of arcs. Type-I arcs emanate from regions on the first time period, and represent deterministic information in the form of known loads and empty movements. Known loads are modelled as links where the "cost" is the net revenue earned from the load, and have an upper bound given by the number of loads available at that price. If several loads are available at different prices, individual arcs can be generated between the same pair of regions, each with a separate cost reflecting the cost and revenues attributed to that particular load. Empty movements are modelled as links with negative costs equal to the cost of the empty move and no upper bounds.

Type-II arcs, also referred to as the "stochastic" arcs, emanate from each region at each point in time and terminate at a dummy supersink node. These arcs are used to summarize the output of the regional impact model which estimates the value of an additional vehicle sent to a region, loaded or empty. In addition, type-II arcs emanating from each region in the first time period are used to summarize the value of holding a vehicle in the same region until later in the day. An illustration of a more detailed set of links is shown in Fig. 3 for a hypothetical region and time period. The figure consists of five links, the first four having an upper bound of one and the last one having an unlimited upper bound which serves as a kind of sink. The "costs"

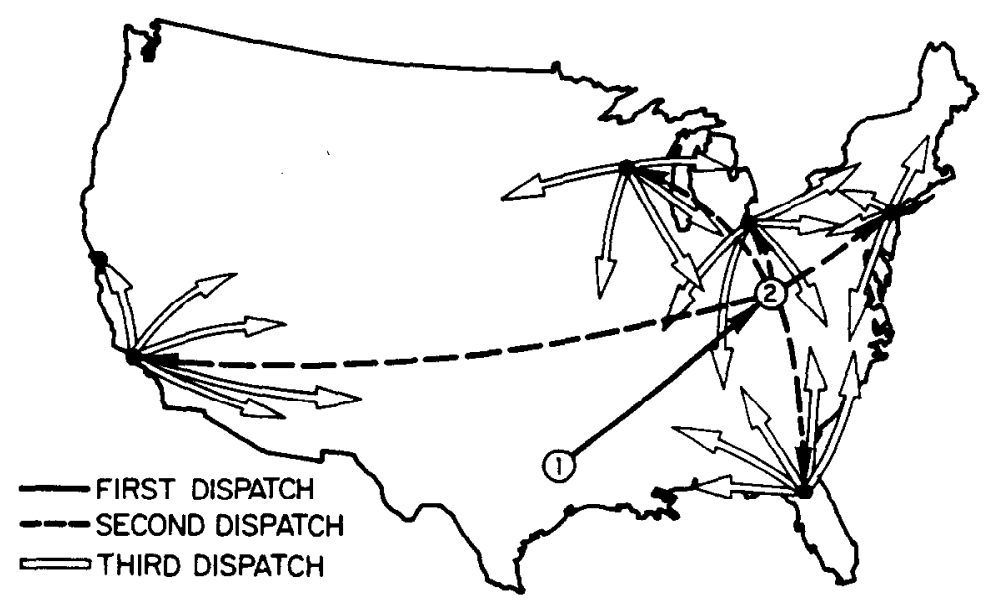

Fig. 1. Illustration of the uncertain future trajectory of a vehicle. 


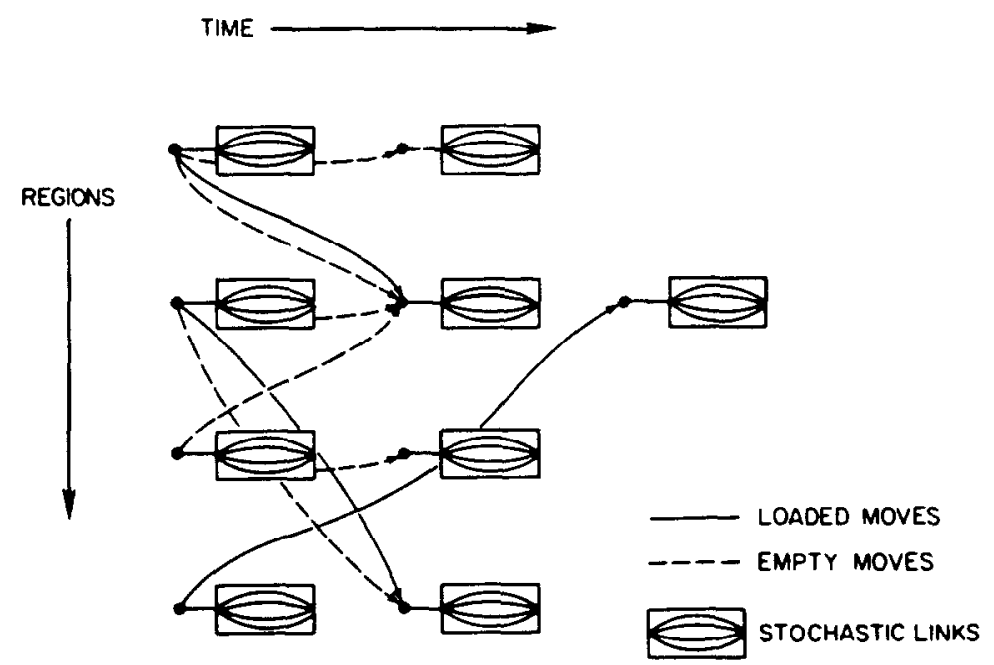

Fig. 2. Initial stochastic network with known loads in the first time period.

on each link are sorted from the most positive to the most negative, and can be interpreted as the total relative value of each vehicle sent to a region. This value includes the expected earnings of a vehicle beginning with the first movement out of the region (which is the second dispatch of the vehicle when we measure from the first time period) as well as total subsequent earnings until the end of the planning horizon. As each additional vehicle is sent into a region, the marginal contribution declines until some minimum contribution (or maximum loss) is reached.

The set of type-II arcs emanating from a region at some point in the future represent the output of the regional impact model, and the calculation of the values used on these arcs is detailed in Sections 2, 3 and 4. Assuming that these numbers are available (and their calculation does require some approximations), the general structure of the model beings to become clear. Links emanating from regions in the first time period contain real-time information about currently available loads as well as possible empty movements. The decision to move a vehicle along one of these links can be modelled as a deterministic process. Once a vehicle arrives in a region at some point in the future (typically two to four days), the opportunities available are uncertain and hence it is no longer possible to track the vehicle in a deterministic manner as would be required by White (1972) or Powell et al. (1984). Instead, the regional impact model is used to represent the marginal value of each additional vehicle sent to a region at some point in the future.

With the structure of the model in mind, it is possible to provide additional insights into

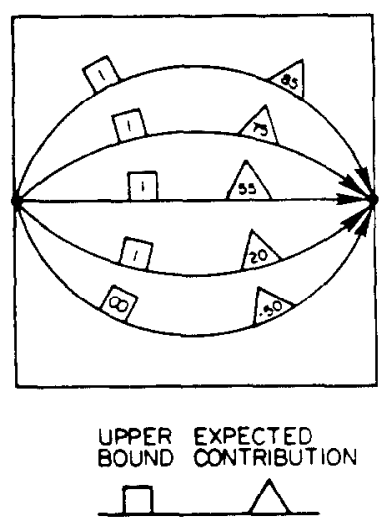

Fig. 3. Detailed illustration of stochastic links summarizing the regional impact model. 
the logic behind the modelling approach. It is important when dispatching to understand the marginal value of an additional vehicle in each region at each point in time. It is possible, using the regional impact model, to find the value of an additional vehicle in each region, but only at the end of the first stage of the trajectory is the number of vehicles in each region actually known with certainty. Since the activity of each vehicle in the second (and future) stages is uncertain, the number of trailers in each region at these future points in time is uncertain. The ability to model such uncertain trajectories is simply outside the limitations of a pure network model. For this reason, future earnings during the third stage will be modelled as a constant per vehicle, independent of the number of vehicles in each region. If the trajectory of a vehicle were modelled using only two stages, namely the first deterministic move followed by all future stochastic moves, it would be necessary to model the value of an additional vehicle at the end of the first stage (that is, at the end of the first deterministic move) as a constant, which would could create situations where there are far too many or too few vehicles in certain regions.

The important feature of the network structure being used is that once the costs and upper bounds on each link are computed, the entire problem can be optimized using an efficient network simplex algorithm which is not only computationally very fast but also provides integer solutions which can be directly implemented.

The network structure displayed in Fig. 2 is a standard one for problems of this type. Cooper and Leblanc (1972) consider a problem with similar structure of a transportation problem with uncertain demands and stockout costs. The uncertain demands can be represented as nonlinear link costs producing a convex nonlinear network problem that can be solved using either a nonlinear network algorithm such as Frank-Wolfe or using a piecewise linear approximation, producing the network in Fig. 2. Cooper and Leblanc presented experiments supporting the conclusion that the Frank-Wolfe algorithm is faster, but their solution of the piecewise linear approximation used an out-of-kilter code which is typically at least an order of magnitude slower than current simplex codes. Aside from simple speed, the integrality of the vehicles implies that for our problem the value of an additional vehicle sent into a region is indeed a piecewise linear function.

\section{THE REGIONAL IMPACT MODEL}

The regional impact model estimates the value of an additional vehicle sent into a region. To perform this function it must attempt to approximate the decision process that will determine what will happen to a vehicle once it arrives in a region on a given day. The following data are assumed to have been provided based on past historical and future expected performance:

$$
\begin{aligned}
F_{j}(i, s)= & \text { random variable denoting the forecasted number of vehicles that will be moved } \\
& \text { full from region } i \text { to } j \text { on day } s \\
E_{j}(i, s)= & \text { random variable denoting the forecasted number of vehicles that will be moved } \\
& \text { empty from region } i \text { to } j \text { on day } s, \\
r_{i, j}= & \text { net revenue per loaded move from region } i \text { to } j, \\
c_{i, j}= & \text { cost per empty move from } i \text { to } j, \\
p_{j}(s)= & \text { average total net revenue a vehicle arriving in region } j \text { on time period } s \text { will earn } \\
& \text { until the end of the planning horizon, } \\
P= & \text { last period in the planning horizon, } \\
t_{i, j}= & \text { travel time (in integer periods) from } i \text { to } j \text { (for simplicity, empty movements are } \\
& \text { assumed to take the same length of time as a full movement). }
\end{aligned}
$$

The expected flows of fulls and empties can generally be developed based on a combination of past historical data and a time series forecasting model. The need to forecast empties should seem a little odd; the empties play a somewhat heuristic role in the model which is described below. The probability distributions of the number of fulls and empties moving to each region can be constructed directly from several months of past data or may be a fitted distribution based on perhaps just the average number of fulls and empties that have moved over the last several months. The easiest approach is to use forecast the means of $F_{j}(i, s)$ and $E_{j}(i, s)$ (using a time series model) and then to fit a Poisson distribution based on these means. The use of the Poisson 
can be justified in situations where the demands are drawn from a large population. In some situations, however, loads in a particular region may be drawn from a small number of large shippers. Data were obtained from a large national carrier giving the frequency of shipments hauled in each traffic lane (region to region pair). Chi-squared goodness of fit tests were run on each distribution at a 95\% confidence level to determine the validity of the Poisson assumption, and only $3 \%$ of the traffic lanes (out of approximately 3200 ) failed the test. Instances where the test failed occurred in the traffic lanes with the heaviest flows which exhibited a bimodal distribution with a much higher probability of little or no flow than would be predicted by the Poisson.

The regional impact model works as follows. To approximate the decision making process in a given region $i$ at time $s$, assume that each possible way of using a vehicle (sending it loaded or empty to each region $j$ ) is evaluated by adding the net revenue earned on the first move out of the region ( $r_{i, j}$ if the vehicle moves full and $-c_{i, j}$ if the vehicle moves empty) to the average net revenue it will earn once it arrives in region $j$ until the end of the planning horizon, given by $p_{j}\left(s+t_{i, j}\right)$. To understand the nature of this approximation, define:

$S(j, s)=$ the total number of vehicles that will be in region $j$ at time $s$,

$\phi_{j}[s, S(j, s)]=$ the marginal value of another vehicle in region $j$ at time $s$, given that the total number of vehicles in the region is $S(j, s)$.

Ideally, the impact of sending another vehicle, say, loaded into region $j$ would be $r_{i, j}+\phi_{j}[s+$ $\left.t_{i, j}, S\left(j, s+t_{i, j}\right)\right]$. The important approximation being made in this model is that we are making the assumption that $\phi_{j}[s, S(j, s)]=p_{j}(s)$, where $p_{j}(s)$ of course is constant with respect to $S(j, s)$. The implication of using a constant marginal contribution function for the downstream impact of another vehicle, instead of the nonlinear function $\phi_{j}[s, S(j, s)]$, is that we will be able to optimize dispatching decisions out of regions at future points in time without considering jointly the decisions being made in other regions. Furthermore, $\phi_{j}[s, S(j, s)]$ is being approximated hy the historical average contribution of another vehicle in region $j$ at time $s$.

Denote the range of possible loaded and empty moves as the dispatch options available out of a region. Defining $w_{n}(i, s)$ to be the estimated value of the $n^{\text {th }}$ option outbound from region $i$ at time $s$, we may write

$$
\begin{aligned}
& \text { If } s+t_{i, j}<P: \quad w_{n}(i, s)= \begin{cases}r_{i, j}+p_{j}\left(s+t_{i, j}\right) & \text { if the } n^{\text {th }} \text { option is to send the } \\
& \text { vehicle full to region } j \\
-c_{i, j}+p_{j}\left(s+t_{i, j}\right) & \text { if the } n^{\text {th }} \text { option is to send the } \\
\text { vehicle empty to region } j,\end{cases} \\
& \text { If } s+t_{i, j} \geq P: \quad w_{n}(i, s)= \begin{cases}r_{i, j}\left[(P-s) / t_{i, j}\right] & \text { if the } n^{\text {th }} \text { option is to send } \\
-c_{i, j}\left[(P-s) / t_{i, j}\right] & \text { the vehicle full to region } j\end{cases} \\
& \text { if the } n^{\text {th }} \text { option is to send } \\
& \text { the vehicle empty to region } j .
\end{aligned}
$$

Equation (1a) states that the average value of a vehicle used on option $n$ is the revenue or cost from the immediate activity (moving loaded or empty) plus the end effect from arriving in destination region $j$ at time $s+t_{i, j}$. If $s+t_{i, j} \geq P$, then eqn ( $1 \mathrm{~b}$ ) allocates that portion of the costs or revenues incurred within the planning horizon to the move.

Finally assume that the options have been ranked so that $w_{1}(i, s) \geq w_{2}(i, s) \geq \cdots \geq$ $w_{N}(i, s)$, where $N$ is the total number of options being considered. Typically, the $N^{\text {th }}$ option is a "sink" which is not necessarily associated with a move to a particular destination. The value of this sink option will be denoted $-C$, where $C$ is a suitably chosen number approximately representing the worst a vehicle could earn. No option with a value worse than $-C$ will be considered.

The regional impact model approximates the dispatching process by assuming that as many vehicles are used on option 1 as is possible, after which as many vehicles are used on option 2 as is possible, and so on until all available vehicles have been used. The question now is determining how many vehicles can be used for each option. If option $n$ is a loaded option, then 
clearly we cannot send any more vehicles than there are loads available. If an option represents an empty move to region $j$, however, there is in principle no limit to how many vehicles could be used, implying that the highest ranked empty option would also be the lowest ranked option that was used by any vehicles. This, however, would be taking advantage of the fact that we are not accurately modelling the function $\phi_{j}[s, S(j, s)]$, but rather are using a constant to model the impact of an additional vehicle in the destination region.

This problem can be handled in the following manner. First define the following random variable:

$$
\begin{aligned}
X_{n}(i, s)= & \text { the maximum allowable number of vehicles that may be dispatched using the } \\
& n^{\text {th }} \text { option out of region } i \text { at time } s .
\end{aligned}
$$

The key to the regional impact is the definition of the random variable $X_{n}(i, s)$. We are going to require that the actual number of vehicles dispatched using the $n^{\text {th }}$ option (which is random) to be less than or equal to $X_{n}(i, s)$. If option $n$ is a loaded move to region $j$, then we will use $X_{n}(i, s)=F_{j}(i, s)$ to reflect the fact that we cannot send any more vehicles loaded than there are loads available. $X_{n}(i, s)$ must be a random variable because $F_{j}(i, s)$ is random. Alternatively, if option $n$ represents an empty move, then a natural choice is to let $X_{n}(i, s)=E_{j}(i, s)$. This definition of $X_{n}(i, s)$ for empty moves is clearly heuristic in nature but greatly simplifies the model. Encouragingly, numerical results suggest that the results of a solution are not particularly sensitive to the use of empty frequencies in this way. Note that for the $\operatorname{sink}$ option $N$ we always use $X_{N}(i, s)=\infty$.

What we are interested in is the expected contribution the $k^{\text {th }}$ vchicle in the region will contribute. Since the maximum number of vehicles that can be used on any one option is a random variable, it is possible that the $k^{\text {th }}$ vehicle could be used for any one of a number of options. Define:

$$
\begin{aligned}
d_{k, n}(i, s) & =\text { the probability the } k^{\text {th }} \text { vehicle in region } i \text { at time } s \text { is used on option } n \\
v_{k}(i, s) & =\text { expected average contribution of the } k^{\text {th }} \text { vehicle in region } i \text { at time } s .
\end{aligned}
$$

The set $\{d(i, s)\}$ is referred to as the dispatch probabilities, and their calculation is described in Section 3 . The expected contribution of the $k^{\text {th }}$ vehicle is now calculated easily as

$$
v_{k}(i, s)=\sum_{n} w_{n}(i, s) d_{k, n}(i, s)
$$

The next task is to calculate the dispatch probabilities.

\section{THE DISPATCH PROBABILITIES}

The key set of calculations in the model consists of determining the dispatch probabilities. Throughout this section assume we are dispatching vehicles out of region $i$ at time $s$, and hence the designation $(i, s)$ will be dropped. Let

$$
x_{k}(n)=\operatorname{Prob}\left[X_{n}=k\right]
$$

be the distribution of the random variable $X_{n}$. Next define:

$$
\begin{aligned}
Y_{n} & =\text { total number of allowable moves to options } 1,2, \ldots, n \\
& =\sum_{k=1}^{n} X_{k}, \\
y_{i}(n) & =\operatorname{Prob}\left[Y_{n}=i\right] .
\end{aligned}
$$

In words, the probability the $k^{\text {th }}$ vehicle is dispatched along option $n$ is equivalent to the joint probability $Y_{n-1}$ is less than or equal to $k-1$ and that $Y_{n}$ is greater than or equal to $k$. 
Table 1. Opportunities for using vehicles outbound from region 3 time period 1

\begin{tabular}{|c|c|c|c|c|c|c|c|c|c|c|c|}
\hline \multirow{2}{*}{\multicolumn{2}{|c|}{$\begin{array}{l}\text { Dest. } \\
\text { reg/time }\end{array}$}} & \multirow{3}{*}{$\begin{array}{c}\begin{array}{c}\text { Net rev. } \\
\text { from move }\end{array} \\
125\end{array}$} & \multirow{3}{*}{$\frac{\text { type }^{\prime}}{\mathrm{L}}$} & \multirow{3}{*}{$\begin{array}{r}\begin{array}{c}\text { End } \\
\text { effect }\end{array} \\
160\end{array}$} & \multirow{3}{*}{$\begin{array}{c}\begin{array}{c}\text { Mean } \\
\text { forecast }\end{array} \\
.5\end{array}$} & \multicolumn{6}{|c|}{ Distribution of available loads ${ }^{2}$} \\
\hline & & & & & & \multirow{2}{*}{$\frac{0}{.606}$} & \multirow{2}{*}{$\frac{1}{.303}$} & \multirow{2}{*}{$\frac{2}{.076}$} & \multirow{2}{*}{$\frac{3}{.014}$} & \multirow{2}{*}{$\frac{4}{0}$} & \multirow{2}{*}{$\frac{5}{0}$} \\
\hline 1 & 3 & & & & & & & & & & \\
\hline 4 & 3 & 80 & $\mathrm{~L}$ & 175 & 1.6 & .202 & .323 & .258 & .138 & .055 & .018 \\
\hline 2 & 5 & 190 & L & 20 & 1.2 & .301 & .361 & .217 & .087 & .026 & .008 \\
\hline 1 & 2 & -210 & $\mathrm{E}$ & 175 & .2 & .819 & .164 & .016 & .001 & 0 & 0 \\
\hline 3 & 2 & 0 & $\vec{S}$ & -100 & & & & & & & \\
\hline
\end{tabular}

' $\mathrm{L}=$ loaded movement; $\mathrm{E}=$ empty movement; $\mathrm{S}=$ sink option.

'Probability distribution fitted from Poisson.

Mathematically, this probability is then

$$
d_{k, n}=\operatorname{Prob}\left[Y_{n-1}<k, Y_{n} \geq k\right] .
$$

The right-hand side of (4) may be easily rewritten to yield

$$
\begin{aligned}
d_{k, n}= & \operatorname{Prob}\left[Y_{n-1}<k\right]+\text { Prob }\left[Y_{n} \geq k\right] \\
& -\operatorname{Prob}\left[Y_{n-1}<k \text { or } Y_{n} \geq k\right] .
\end{aligned}
$$

Since $Y_{n} \geq Y_{n-1}$, the last probability on the right-hand side of (5) must always be identically equal to one, yielding

$$
d_{k, n}=\operatorname{Prob}\left[Y_{n-1}<k\right]-\operatorname{Prob}\left[Y_{n}<k\right] .
$$

The probability mass function of the random variable $Y_{n}$ can of course be easily calculated by convolving the distributions of $X_{1}, X_{2}, \ldots, X_{n}$ together, using the assumption that these distributions are independent. If, however, the random variables $X_{n}$ are assumed to follow a Poisson distribution, then $Y_{n}$ also follows a Poisson distribution.

To illustrate the calculation of the dispatch probabilities, consider a region that has five potential options for using a vehicle. The relevant data is shown in Table 1 which includes the net revenue (which may be negative if the move is empty) from the initial dispatch, the region and time period the vehicle will arrive and the average net revenue the vehicle will earn once it arrives in the destination region until the end of the planning horizon. In addition, the historical distribution of the number of vehicles used for each option is also shown (each distribution was found by using the average number of vehicles forecasted for the option and then fitting a Poisson distribution). The only exception is for the "sink" option, which has been assigned a value of -100 and for which there is no upper bound.

Using eqns (2) and (6), it is straightforward to show that the dispatch probabilities and expected contributions for each vehicle are as shown in Table 2 . Given the small size of the example, there is some probability (.030) that even the first vehicle will be assigned to the sink. As more vehicles are sent to region $i$ at time $s$, the expected contribution of each vehicle drops

Table 2. Dispatch probabilities and expected net revenue for each vehicle

\begin{tabular}{ccccccc}
\hline & & \multicolumn{5}{c}{ Dispatch probabilities for each option' } \\
\cline { 3 - 7 } Vehicle & $\begin{array}{c}\text { Expected } \\
\text { net revenue }\end{array}$ & 1 & 2 & 3 & 4 & 5 \\
\hline 1 & 250 & .394 & .484 & .086 & .007 & .030 \\
2 & 193 & .090 & .530 & .221 & .023 & .136 \\
3 & 117 & .014 & .336 & .290 & .039 & .321 \\
4 & 40 & 0 & .161 & .258 & .044 & .537 \\
5 & -21 & 0 & .062 & .175 & .037 & .725 \\
6 & -61 & 0 & .020 & .097 & .025 & .858 \\
7 & -83 & 0 & .006 & .045 & .014 & .935 \\
\hline
\end{tabular}

$' 1=$ loaded to region $1 ; 2=$ loaded to region $4 ; 3=$ loaded to region $2 ; 4=$ empty to region $1 ; 5=$ sink. 
and the probability that a vehicle will have to be dispatched empty out of the region, or held over to the next day, increases quickly. In actual examples, there might be 30 or 40 possible outbound options, and the probability of putting a vehicle in the sink option remains insignificant until the region becomes saturated. The overall methodology is one which seems to work well for large problems but which does not look realistic in the context of small test problems.

At this point, the last task is to find the regional end effects which give the average net revenue earned by a vehicle beginning with the third dispatch until the end of the planning horizon. This is described in the next section.

\section{REGIONAL END EFFECTS}

The average net revenue earned by a vehicle beginning at a certain point in time until the end of the planning horizon can be found by viewing the movement of a vehicle over time as a nonstationary, but cyclical, Markov chain. The calculation of the end effects can be easily carried out by effectively simulating the movement of vehicles forward in time. The probabilistic trajectories of these vehicles will be based purely on historical data. As before, define a set of options representing the possible loaded and empty moves out of region $i$ at time $s$, and define:

$$
\begin{aligned}
& u_{n}(i, s)=\text { average number of vehicles historically used for the } n^{\text {th }} \text { option, } \\
& \theta_{n}(i, s)=u_{n}(i, s) / \sum_{k} u_{k}(i, s)
\end{aligned}
$$

and as before let $w_{n}(i, s)$ be the average value of an additional vehicle being used for the $n^{\text {th }}$ option. If option $n$ represents moving a vehicle full from region $i$, time $s$ to region $j$, arriving at time $s+t_{i, j}$, then $u_{n}(i, s)$ is the average number of vehicles forecasted to move loaded in this lane at this time, and the value would be $w_{n}(i, s)=r_{i, j}+p_{j}\left(s+t_{i, j}\right)$. The fractions $\theta_{n}(i, s)$ can be interpreted as an estimate of the probability that a randomly chosen vehicle in region $i$ at time $s$ will be used for option $n$.

With these definitions in hand, the region end effect values $p_{i}(s)$ are easily calculated with the following recursion:

$$
\begin{aligned}
p_{j}(P) & =0 \quad \forall j, \\
p_{j}(s) & =\sum_{n} \theta_{n}(j, s) w_{n}(j, s) \quad \forall j, s=1,2, \ldots, P-1,
\end{aligned}
$$

where the values $w_{n}(j, s)$ are as defined using eqn (1). Equation (8a) simply expresses a boundary condition that we are disregarding any revenues generated after the end of the planning horizon. The remaining end effects $p_{j}(s)$ are then easily calculated using (8b) by beginning with $s=P$ 1 , and proceeding backwards in time until all regions have been covered for all points in time. This process is extremely fast, allowing the use of rcasonably long planning horizons.

This approach is a very simple way of simulating a nonstationary Markov chain. To develop an appreciation of what the end effects $p_{j}(s)$ mean, consider a simple system where all interregional travel times are exactly one day, and all vehicles either move loaded between regions or they are held over in the same region until the next day. Further assume that the pattern of loads out of every region does not vary from one day to the next. Under these conditions, we can view the movements of vehicles over time and space as a first order, stationary Markov chain with a probability transition matrix defined by

$$
\mathbf{A}=\left\|\boldsymbol{\alpha}_{i, j}\right\|,
$$

where

$$
\alpha_{i, j}=\left\{\begin{array}{l}
\text { probability of moving loaded from } i \text { to } j, j \neq i \\
\text { probability of being held over in } i, i=j
\end{array}\right.
$$


The probabilities $\{\alpha\}$ can be estimated based on historical patterns of loaded movements. Denote the probability state vector by $\pi(s)=\left(\ldots, \pi_{j}(s), \ldots\right)$, where $\pi_{j}(s)$ is the probability a vehicle is in state $j$ at time $s$, and where $\pi(0)$ is the initial state vector for a vehicle. To determine the expected earnings a vehicle will generate over time, let $r_{i, j}$ be the revenue eamed on a load carried from $i$ to $j$, and let $r_{i, i}$ be the (negative) cost of holding a vehicle in a region from one day to the next. We can now define a conditional reward vector $\rho$ (defined as a row vector) as follows:

$$
\rho_{i}=\sum_{j} r_{i, j} \alpha_{i, j}
$$

where $\rho_{i}$ can be interpreted as the expected earnings of a vehicle in region $i$ on its next move. If we let the vector $e_{j}$ denote a (row) vector of zeroes with a one in the $j^{\text {th }}$ component, then the regional end effect $p_{j}(s)$ can be calculated as

$$
p_{j}(s)=e_{j} \sum_{l=s}^{P} \mathbf{A}^{l-s} \boldsymbol{\rho}^{T}
$$

If the matrix $\mathbf{A}$ is irreducible and aperiodic, which it will always be in practice, then it is well known that the limit of the right-hand side of (11) as $P \rightarrow \infty$ has the general form (see, for example Bhat, pp. 339-342)

$$
p_{j}(s)=\beta_{j}+\delta(P-s)
$$

for large values of $P$. In other words, if we follow this vehicle far enough into the future, then its earnings will grow linearly in a way that does not depend on the starting region $j$. The constant $\beta_{j}$, on the other hand, carries the initial effect of starting in region $j$.

Assume now that we have chosen a planning horizon $P$ large enough so that eqn (12) is a reasonable approximation of the regional end effect values for periods $s$ "close" to the beginning of the planning horizon (typically we are interested in only the first four or five time periods). In this case, increasing $P$ by one has the effect of increasing every $p_{j}(s)$ by exactly $\delta$, which will have no impact on any dispatching decisions. Assume we are interested in $p_{j}(s)$ for $s=1, \ldots$, $P^{\prime}$, where $P^{\prime}$ should be appreciably smaller than $P\left(P^{\prime}\right.$ is the latest time period for which we have to generate type-II links in the network in Fig. 2). We could choose a region $J$ at a particular point in time $T$ and use this as a base value, and then redefine each end effect using $P_{j}^{\prime}(s)=$ $p_{j}(s)-p(T)$ without affecting any of the dispatching decisions. For this reason, the end effects $p_{j}(s)$ are best viewed as the relative value of another vehicle in region $j$ at time $s$, and as a result the absolute value of $p_{j}(s)$ has little practical value.

An obvious question to ask at this point is exactly how far into the future we need to go before (12) becomes a reasonable approximation of the total contribution earned by a vehicle starting in region $j$. An experiment was conducted using an actual data set with 60 regions, where the end effects $p_{j}(s)$ were found for a group of regions for planning horizons of varying lengths. The dispatch probabilities $\theta_{n}(i, s)$ were calculated using historical loaded and empty flows that varied by day of week. The relative end effect $p_{j}^{\prime}(s)$ was defined using $p_{j}^{\prime}(s)=p_{j}(s)-$ $p_{1}(1)$, and the value $p_{j}^{\prime}(1)$ was plotted for three candidate regions for planning horizons ranging from 1 to 20 days. Note that since the dispatch probabilities varied by day of week, the linear function depicted in eqn (12) will never properly hold true if time $t$ is measured in days (it will hold if $t$ is measured in integer weeks, since we did assume that the flows were repeated from one week to the next). Instead, the limiting form of $p_{j}(s)$ for large $P$ would be given by

$$
p_{j}(s)=\beta_{j}+\delta(P-s)+\Psi(\bmod [s, 7]+1),
$$

where $\Psi(t), t=1,2, \ldots, 7$, is a function which describes a correction reflecting the effect of the day of the week. A formal derivation of (13) is not given here, but its proof is easily seen by first considering the process from one Monday to the next, and then considering what must 


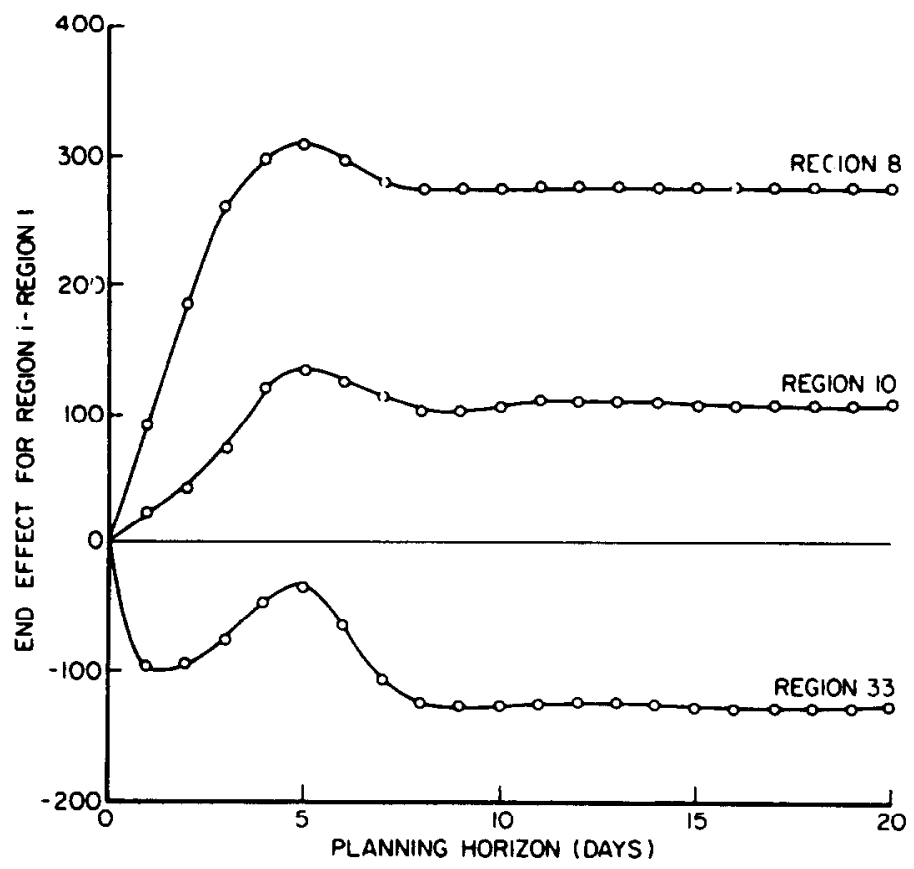

Fig. 4. Convergence of relative end effects of planning horizon is increased.

occur between Mondays. However, it can be easily seen that the function $\Psi(t)$ has no effect on $p_{j}^{\prime}(s)$, and as $P$ becomes large, $p_{j}(1)$ will approach a constant that approximates $\beta_{j}-\beta_{1}$.

The results are shown in Fig. 4, which suggests that the process has reached steady state in approximately 10 days. These results were typical of all the regions, which demonstrated very similar behavior. Thus if the stochastic links are generated as far as, say, five days into the future, then a planning horizon of 15 days should be used.

At this point, we have finished all the calculations required to generate the network shown in Fig. 2. The next section extends the basic network model in an important way, after which Sec. 6 describes how to forecast future supplies of vehicles in each region.

\section{THE EXTENDED NETWORK MODEL}

The network shown in Fig. 2 is restrictive in one very important way, namely that it does not appear to allow known loads to be called in for time periods in the future. Although a relatively small proportion of the total loads are called in one or more days in advance, it is important for a shipper to be able to call in loads for tomorrow or the next day, and the model should be able to explicitly recognize this load and respond to it. In fact, loads which need to be picked up in the future are represented by simply introducing deterministic arcs at the appropriate points in time. A more realistic view of the network that would result with a mixuture of loads to be picked up both today and several days into the future is illustrated in Fig. 5 . Deterministic arcs, representing both loaded and empty moves, can in principle be introduced at any point in time. Normally, however, a given region will be represented only as far into the future as is needed to represent known loads, or empty repositioning moves to known loads out of other regions.

Earlier, the trajectory of a vehicle was described as comprising of three phases: the first "deterministic" dispatch, the second (uncertain) dispatch, and then all remaining (uncertain) dispatches. The network in Fig. 5 suggests that while the trajectory of a vehicle can indeed be described as comprising three phases, these phases are better described as follows. The first, deterministic phase consists of one or more deterministic (loaded or empty) moves which follow the vehicle as far into the future as it can be tracked deterministically. Once it lands in a region where it must be used in an uncertain way, there is the second phase consisting of the first 


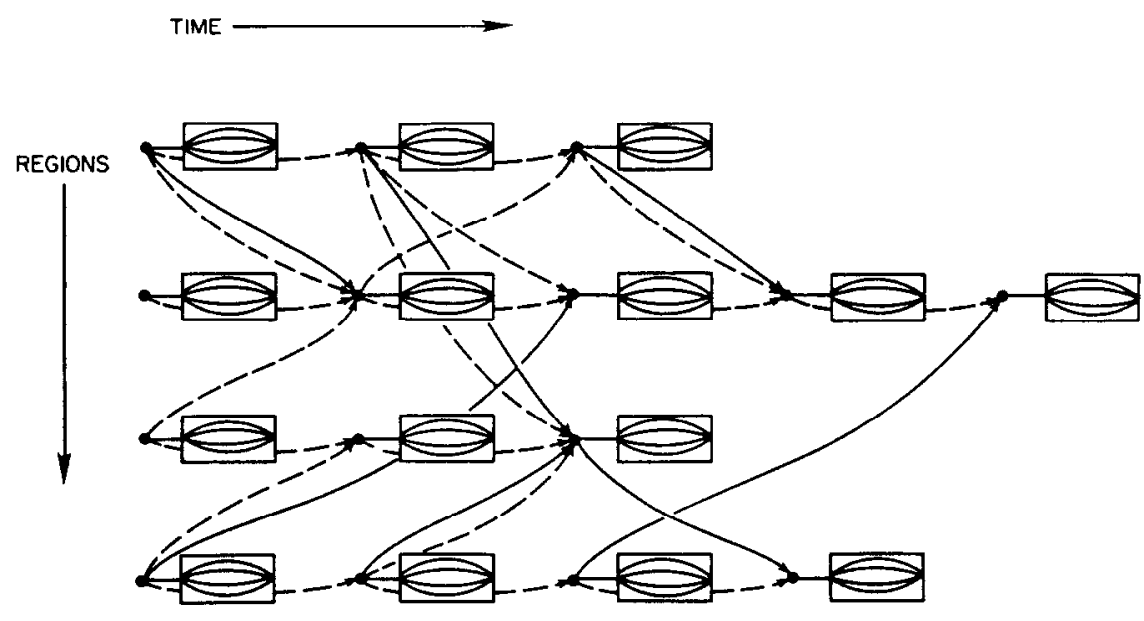

Fig. 5. Extended stochastic network model with known loads in future time period.

random dispatch. Finally, the third phase begins with the second random dispatch until the end of the planning horizon. In this way, it is possible for the model to recommend that a vehicle be moved empty into another region in order to be in position to handle a known load tomorrow. The attractiveness of the approach is that if it becomes possible to know with greater certainty loads farther into the future, the model responds by modelling "known" information as far into the future as it is available, after which point the "stochastic" part takes over.

\section{ESTIMATING FUTURE VEHICLE SUPPLIES}

Even using realistic data, the model can typically track the trajectory of most vehicles for up to a few days before it is forced into a stochastic link. Once this occurs, the future path of the vehicle is modelled only approximately, and is not represented explicitly within the network. This is particularly true of vehicles which are routed into the stochastic links for the first time period, which estimates the value of holding a vehicle in a region until later in the day. What this process implies is that the total number of vehicles arriving in regions on a given day in the future can be substantially less than the total fleet size. Beyond a certain point in time, a region may not be explicitly represented at all in the network.

It is often the case that a carrier would like to estimate, if only approximately, the total number of vehicles that will be in a region five or more days into the future. After the network model has been run, it is possible to track the future stochastic paths of vehicles arbitrarily far into the future by using the different dispatch probabilities for vehicles. For the purposes of this section, let $S(i, s)$ be the expected number of vehicles in region $i$ at time $s$. In addition, define:

$$
\begin{aligned}
R(i, s)= & \text { the number of vehicles dispatched prior to the beginning of the planning horizon } \\
& \text { that are becoming available in region } i \text { at time } s \text { (this quantity is an input to the } \\
& \text { model), } \\
Q(i, s)= & \text { expected number of vehicles in region } i \text { at time } s \text { which are being dispatched } \\
& \text { "stochastically," } \\
g(i, s, j)= & \text { number of vehicles dispatched deterministically (loaded or empty) from region } \\
& \left.i, \text { time } s \text { to region } j \text { (arriving at time } s+t_{i, j}\right), \text { in the optimal network solution, } \\
h(i, s)= & \text { number of vehicles allocated to the stochastic links in region } i \text {, time } s, \text { in the } \\
& \text { optimal network solution [let } h(i, s)=0 \text { if no stochastic links were generated } \\
& \text { for region } i \text {, time } s] .
\end{aligned}
$$

In the above notation, the vectors $\{g(i, s, j)\}$ and $\{h(i, s)\}$ summarize the optimal solution returned after the network in Fig. 5 has been solved. The problem now is to forecast the future trajectories of the vehicles assigned to each set of stochastic links. This can be done by recursively finding the expected number of vehicles in each region $i$ at each point in time, $S(i, s)$, beginning with 
the first time period and then working forward in time. Any vehicles that have not been dispatched deterministically by the model will be governed by the dispatch probabilities $\left\{d_{k, n}(i, s)\right\}$ described in Section 3. It is convenient, however, to introduce a slightly modified notation. For a given region $i$ at time $s$, let $n$ be the option representing moving loaded from $(i, s)$ to $j$, and let $m$ be the option representing moving empty to region $j$, and define:

$$
\hat{d}_{k, j}(i, s)=d_{k, n}(i, s)+d_{k, m}(i, s),
$$

where $\hat{d}_{k, j}(i, s)$ represents the probability that the $k^{\text {th }}$ vehicle will be dispatched (loaded or empty) to region $j$, arriving at time $s+t_{i, j}$. The overall procedure is given as follows:

Step 1: $\quad$ Set $S(i, s)=R(i, s)$ for all $i$ and all $s$; Set $Q(i, s)=h(i, s)$ for all $i$ and all $s$;

Set $s=1$.

Step 2: Set $s=s+1$.

Step 3: For all regions $i$ and for all regions $j$ where $s+t_{i, j}<P$ : set

$$
\begin{aligned}
& Q\left(j, s+t_{i, j}\right)=Q\left(j, s+t_{i, j}\right)+\sum_{k=1}^{\mid Q(i, s)]} \hat{d}_{k, j}(i, s) \\
&+\{Q(i, s)-[Q(i, s)]\} \hat{d}_{[Q(i, s)]+1, j}(i, s)
\end{aligned}
$$

and set

$$
S\left(j, s+t_{i, j}\right)=S\left(j, s+t_{i, j}\right)+Q\left(j, s+t_{i, j}\right)+g(i, s, j),
$$

where $[x]$ is the largest integer less than or equal to $x$.

Step 4: If $s<P-1$, go to Step 2.

The last term on the right-hand side of $(15)$ is designed to handle the situation where $Q(i, s)$ is fractional. If, for example, $Q(i, s)=10.7$, the first 10 vehicles are dispatched according to the first 10 dispatch probabilities, while the last .7 of a vehicle is dispatched according to the dispatch probability for the eleventh vehicle.

This recursion is a simple way of finding the expected number of vehicles in each region at each point in time, taking account of both deterministic moves dictated by the network model as well as the dispatch probabilities governing vehicles whose trajectories are uncertain. Note that in this procedure, whereas the network model in Fig. 5 might indicate that, for example, 10 vehicles are being dispatched stochastically in region 12 at time period 5 , the actual expected supply of vehicles being dispatched stochastically out of 12 at time 5 might be greater than 10 due to vehicles dispatched stochastically out of other regions at earlier time periods.

An interesting enhancement of this simulation would be to actually calculate the distribution of the number of vehicles in each region at each point in time. By making appropriate independence assumptions about the supplies of vehicles in different regions, this problem could be solved easily by finding the distribution of vehicles being dispatched in each lane, and then convolving this distribution into the distribution of vehicles already in the destination region. It is unclear, however, whether or not the full distribution would add significantly to our understanding of the future behavior of the network.

\section{THE EFFECT OF UNCERTAIN SUPPLIES OF VEHICLES ON DOWNSTREAM IMPACTS}

The most important theoretical weakness of the basic modelling approach is its inability to directly incorporate the effect of uncertain future supplies of vehicles on vehicles being dispatched now. The network model can track vehicles only as far as their paths are known deterministically. Once a vehicle must be dispatched in an uncertain way, the model no longer tracks the vehicle 
explicitly. This creates two sources of errors. First, the supply of vehicles for each region $i$, time period $s$, will be underestimated to a degree that increases with $s$. Thus, a given region may normally have 20 vehicles, but on day 5 there may be only 3 vehicles whose trajectories have been deterministically tracked to this region. The value of an additional vehicle in this region on day 5 , then, may seem very high since vehicles which may eventually end up there have not been accounted for.

The second error relates to the use of an average value of another vehicle in a region as a measure of the end effect. The methodology described in Section 4 does not even attempt to estimate the marginal contribution of an additional vehicle, since the total number of vehicles that will be in each region is not known in advance.

An argument can be made with regard to the first "error" that in fact we do not want to take into account uncertain supplies of vehicles. Assume that the value of the first five vehicles in region $i$ at time $s$, as represented by the values $v_{k}(i, s)$ are given by $\$ 100, \$ 75, \$ 50, \$ 25$ and $\$ 0$, and that normally there are five vehicles in this region (the assumption is being made that the carrier is actually smart enough to know when the next vehicle would show a loss on a marginal basis). Further assume that there are no deterministic moves into the region, and that tracking the flows on the stochastic links (using, for example, the procedure presented in Section 6) produces an expected supply of five vehicles in the region. Now consider a load that is being booked into region $i$ at time $s$, which is handled by introducing a deterministic (type-I) arc from the origin region into region $i$, time $s$. Depending on the impact of the new known load on flows of "stochastic" vehicles, the marginal value of an additional vehicle in region $(i, s)$ will be approximately $\$ 0$, and the optimization procedure may well reject this load in favor of more lucrative opportunities elsewhere. The model may continue to "reject" loads right up to day $s$, and only then would it finally realize that in fact there are no vehicles actually in the region on day $s$. As presented, the model tries to ensure that at least four vehicles are supplied to the region with only the fifth marginal vehicle being uncertain. In short, the marginal value of a vehicle is being based on known rather than forecasted supplies. This argument is, of course, highly intuitive in nature and needs to be investigated in greater depth on both theoretical and experimental grounds.

Returning to the issue of marginal versus average end effects, it is possible to propose an iterative scheme which may yield good estimates of the marginal contribution of another vehicle in cach region. Define:

$S=$ vector of vehicle supplies for all regions at all points in time,

$=(S(1,1), S(2,1), \ldots, S(j, s), \ldots)$,

$\hat{p}_{j}(s, S)=$ the marginal value of an additional vehicle in region $j$ at time $s$,

$\hat{w}_{n}(i, s)=$ marginal contribution of a vehicle dispatched from region $i$ at time $s$ using the $n^{\text {th }}$ option.

If we knew in advance that there would be $S(j, s)$ vehicles in region $j$ at time $s$, the marginal value of an additional vehicle could be calculated by using the dispatch probabilities for the next vehicle in the region, as follows:

$$
\hat{p}_{i}(s)=\sum_{n} \hat{w}_{n}(i, s) d_{K, n}(i, s)
$$

where $K=[S(i, s)]+1$ denotes the next vehicle to arrive in the region. If option $n$ sends a vehicle to region $j$ and $s+t_{i, j}<P, \hat{w}_{n}(i, s)$ is given by

$$
\hat{w}_{n}(i, s)= \begin{cases}r_{i, j}+\hat{p}_{j}\left(s+t_{i, j}\right) & \text { if the } n^{\text {th }} \text { option is to send the } \\ & \text { vehicle full to region } j \\ -c_{i, j}+\hat{p}_{j}\left(s+t_{i, j}\right) & \text { if the } n^{\text {th }} \text { option is to send the } \\ & \text { vehicle empty to region } j .\end{cases}
$$

Equation (18) uses the fact that the marginal value of a vehicle dispatched from $i$ to $j$ is the direct profit derived from the move $\left(r_{i, j}\right.$ or $\left.-c_{i, j}\right)$ plus the marginal value of the vehicle in the destination 
region $j$ at time $s+t_{i, j}$. If $s+t_{i, j} \geq P$, then $\hat{w}_{n}(i, s)=w_{n}(i, s)$. Using eqns (17) and (18), the marginal end effects $\hat{p}_{j}(s)$ can be calculated by starting at time $s=P$ and working backwards in time. For each region $i$ at time $s$, we are taking the estimated supply of vehicles in that region, $S(i, s)$, and then using the dispatch probabilities to determine what wol. likely happen to the "next" vehicle dispatched to the region. The dispatch probabilities $\left\{d_{k, n}(i, s)\right\}$ are then calculated by ranking the outbound options on the basis of $\hat{w}_{n}(i, s)$ rather than $w_{n}(i, s)$. If this approach were used, a reasonable overall procedure would be as follows.

Step 0: Calculate the average end effects $p_{j}(s)$ and corresponding dispatch probabilities $d_{k, n}(i, s)$ as described in Sections 2,3 and 4.

Set $k=1$.

Step 1: Generate and solve the stochastic network.

Step 2: Simulate the vehicle supplies $S(j, s)$ using the procedure described in Section 6. If $k>1$, then compare these vehicle supplies to those from the previous iteration and test for "convergence." If method has not converged, then proceed to Step 3; otherwise stop.

Step 3: Calculate the marginal end effects $\hat{p}_{j}(s)$ and corresponding dispatch probabilities using eqns (17) and (18).

Step 4: Set $k=k+1$ and go to Step 1 .

This approach is a simple framework which has not been tested. An obvious difficulty is the lack of any guarantee that the process would ever converge, although empirically this may not prove to be a problem. The intent here is simply to demonstrate one possible approach for overcoming a limitation of the basic model which uses only the average end effects $\left\{p_{j}(s)\right\}$. The same basic approach could also be used if we wanted to incorporate forecasted supplies of vehicles on the dispatch probabilities, discussed in the beginning of this section. Recalling that $Q(i, s)$ is the supply of "stochastic" vehicles in region $i$ at time $s$, the number of vehicles in the region would be $Q(i, s)$ plus any additional vehicles dispatched deterministically in the network model. The quantities $Q(i, s)$ would initially be set to zero, and then updated at each iteration of the algorithm. Thus if $Q(i, s)=5$, then the marginal value of the first vehicle in region $(i$, $s)$ would effectively be given by $v_{6}(i, s)$.

\section{DISCUSSION}

As was mentioned in the Introduction, the purpose of this paper has been to describe the basic mechanics of what seems to be a very appealing approach for handling the real time dispatching problem for truckload motor carriers. There are a variety of important issues that have not been covered in this paper, as well as the usual endless list of details that must be addressed when actually implementing a model such as this. This framework is at the heart of a computer code that is currently being implemented at a major truckload carrier, with very encouraging results. This implementation will be described in much greater detail in a separate paper. From a research point of view, it is useful to identify important practical as well as theoretical issues raised by the method.

(1) Among the most important practical problems is the development of accurate forecasts of loads outbound from a region both today and in the future. Many carriers maintain extensive historical databases which can serve as a useful starting point for developing forecasts. The most difficult problem, however, is estimating at $11 \mathrm{a} . \mathrm{m}$. what will happen in a region later the same day, or tomorrow, given the loads that have already been called in.

(2) The use of the end effects $\left\{p_{j}(s)\right\}$ in the regional impact model, as opposed to a more accurate estimate of the marginal value of an additional vehicle, introduces uncertain errors. For realistic problems, it seems unlikely that this approximation will have a large impact on actual dispatching decisions, but it would be useful to evaluate the errors that are being introduced. A corollary to this problem is the development of improved methods for estimating the end effects to more accurately evaluate the marginal impact of an additional vehicle downstream. One 
possibility would be the procedure described in Section 7, although convergence issues would have to be addressed in a more formal way.

(3) The overall model is of course a heuristic approach to avoid enumerating a combinatorially large state space. At this time, no attempt has been made to enumerate what this state space actually is and how one would deal with it, even if only from a theoretical point of view. This problem is currently being investigated by the author and will be reported on in a later paper.

Acknowledgments-The author would like to thank the careful review of two anonymous referees in clarifying the presentation.

\section{REFERENCES}

Bhat U. N. (1984) Elements of Applied Stochastic Processes (2nd ed.). John Wiley and Sons, New York.

Cooper L. and Leblanc L. (1972) Stochastic transportation problems and other network related convex problems. Navel Research Logistics Quarterly 24, 327-337.

Crowe M. J. (1983) Private communication.

Misra S. C. (1972) Linear programming of empty wagon disposition. Rail International 3, 151-158.

Powell W. B., Sheffi Y. and Thiriez S. (1984) The dynamic vehicle allocation problem with random demands, Proceedings of the Ninth International Symposium on Transportation and Traffic Theory (Edited by J. Volmuller and R. Hamerslag), 357-374. VNU Science Press, The Netherlands.

Powell W. B. (1986) A stochastic formulation of the dynamic vehicle allocation problem, Transpn. Sci., 20, 117-129.

White W. W. and Bomberault A. M. (1969) A network algorithm for empty freight car allocation, IBM Systems J. 8, $147-169$.

White W. W. (1972) Dynamic transshipment networks: an algorithm and its application to the distribution of empty containers. Networks 2, 211-236. 